\title{
ESTRATÉGIAS PARA DIMINUIÇÃO DE ERROS DE FORNECIMENTO DE PSICOTRÓPICOS EM UMA UNIDADE BÁSICA DE SAÚDE: RELATO DE EXPERIÊNCIA DE IMPLEMENTAÇÃO
}

\author{
Raissa Pereira Santos ${ }^{1}$ \\ Kaic Leite Meira ${ }^{2}$ \\ Giselly de Almeida dos Santos ${ }^{3}$ \\ Nathallia Resende de Melo Barbosa ${ }^{4}$ \\ Erika Meire dos Santos Martins ${ }^{5}$ \\ Alexandre Vaz Machado ${ }^{6}$
}

RESUMO: Introdução: $O$ acesso aos medicamentos considerados essenciais se estabelece por meio de mecanismos que permitam a contínua atualização da Relação Nacional de Medicamentos Essenciais, imprescindível instrumento de ação do SUS, na medida em que contempla um elenco de produtos necessários ao tratamento e controle das patologias prevalentes no país. No DF, a atual REME AB conta com um elenco de 32 medicamentos que são sujeitos a prescrição de controle especial. É obrigatória a presença do farmacêutico na unidade para o fornecimento desses itens, visto que é atribuição privativa a dispensação, bem como a análise e interpretação do receituário. Entretanto, visto a carência de Especialistas em Saúde, o fornecimento desses medicamentos, por inúmeras vezes, é feito por técnicos administrativos sob supervisão, contribuindo, entre outros motivosm para a incidência de erros de fornecimento. Objetivo: relatar a experiência da elaboração e implementação de estratégias que visam amenizar os erros de fornecimento de medicamentos sujeito a

\footnotetext{
${ }^{\text {I }}$ Farmacêutica Generalista pela Universidade Federal do Maranhão (UFMA) com período sanduiche na Universidad de Valéncia (UV-ES). Residente do Programa Multiprofissional em Saúde da Família e Comunidade - Escola Superior de Ciências da Saúde (ESCS) - DF

${ }^{2}$ Farmacêutico Generalista pela Universidade Federal do Oeste da Bahia (UFOB), Especialista em Farmácia Clínica e Hospitalar, Residente do Programa Multiprofissional em Saúde da Família e Comunidade -Escola Superior de Ciências da Saúde (ESCS) - DF.

3 Farmacêutica Generalista pela Universidade de Brasília. (UNB) Mestre em Ciências Médicas pela Universidade de Brasília (UNB), Residente do Programa Multiprofissional em Saúde da Família e Comunidade -Escola Superior de Ciências da Saúde (ESCS) - DF.

4 Farmacêutica Generalista pela Universidade de Brasília (UNB) Especialista em Saúde da Família e Comunidade - Escola Superior de Ciências da Saúde (ESCS) - DF.

${ }^{5}$ Nutricionista pela Universidade Federal do Maranhão (UFMA) Mestre em Ciências da Saúde pela Universidade Federal do Maranhão (UFMA).

${ }^{6}$ Farmacêutico Clínico Industrial pela Universidade de Brasília (UnB) Especialista em Farmacologia Clínica, Mestre em Ciências Farmacêuticas pela Universidade de Brasília (UnB) Farmacêutico na Secretaria de Estado de Saúde do Distrito Federal.
} 
controle especial. Metodologia: estudo descritivo do tipo relato de experiência a partir da problematização do Arco de Maguerez. Resultados e discussão: notou-se divergência entre o estoque físico e o estoque informatizado de medicamentos psicotrópicos, sendo necessário detectar a origem dos erros e o porquê do acontecimento dos mesmos e, posteriormente, desenvolver estratégias e ferramentas para diminuição da ocorrência dos erros. Dessa forma, foi implementada a dupla checagem durante o fornecimento, elaboração do carimbo lote/validade, contagem diária do estoque físico e estoque informatizado e capacitação dos técnicos administrativos. Conclusão: a implementação dessas medidas se mostrou importante para diminuição dos erros de fornecimento, evitando a ocorrência de possíveis danos aos pacientes ou prejuízos acerca do seu tratamento medicamentoso.

Palavras-chaves: Psicotrópicos. Erros de Medicação. Atenção Primária à Saúde

ABSTRACT: Introduction: Access to medicines considered essential is established through mechanisms that allow the continuous updating of the National List of Essential Medicines, an essential instrument of action of the SUS, as it includes a list of products necessary for the treatment and control of prevalent pathologies in the country. In DF, the current REME AB has a list of 32 drugs that are subject to special control prescription. The presence of the pharmacist in the unit is mandatory for the supply of these items, as the dispensing, as well as the analysis and interpretation of the prescription, is a private attribution. However, given the lack of Health Specialists, the supply of these drugs is often done by administrative technicians under supervision, contributing, among other reasons, to the incidence of supply errors. Objective: to report the experience of designing and implementing strategies that aim to alleviate errors in the supply of medications subject to special control. Methodology: descriptive study of the experience report type from the problematization of the Arch of Maguerez. Results and discussion: there was a divergence between the physical stock and the computerized stock of psychotropic drugs, making it necessary to detect the origin of the errors and why they occurred and, subsequently, develop strategies and tools to reduce the occurrence of errors. Thus, double checking was implemented during delivery, preparation of the batch/validity stamp, daily counting of physical stock and computerized stock, and training of administrative technicians. Conclusion: the implementation of these measures proved to be important to reduce supply errors, preventing the occurrence of possible harm to patients or damage to their drug treatment.

Keywords: Psychotropic Drugs. Medication Errors. Primary Health Care.

\section{INTRODUÇÃO}

No âmbito do Sistema Único de Saúde (SUS), no que tange o acesso integral do usuário ao tratamento, temos a Política Nacional de Medicamentos, que traz como seu propósito precípuo garantir a necessária segurança, eficácia e qualidade dos medicamentos,

a promoção do uso racional e o acesso da população a aqueles que são considerados 
essenciais (BRASIL, 200I).

$\mathrm{O}$ acesso aos medicamentos considerados essenciais se estabelece por meio de mecanismos que permitam a contínua atualização da Relação Nacional de Medicamentos Essenciais, imprescindível instrumento de ação do SUS, na medida em que contempla um elenco de produtos necessários ao tratamento e controle das patologias prevalentes no país (BRASIL, 200I). No âmbito do Distrito Federal (DF), a atual Relação de Medicamentos Essenciais - Atenção Básica (REME-AB) conta com um elenco de 32 medicamentos que são sujeitos a prescrição de controle especial (BRASÍLIA, 202I; BRASIL, 1998).

A farmácia da Unidade Básica de Saúde (UBS) or de Santa Maria realiza o fornecimento de medicamentos de controle especial, constantes na portaria 344/98, desde março de 2019. É obrigatória a presença do farmacêutico na unidade para o fornecimento desses itens, visto que é atribuição privativa do farmacêutico a dispensação, bem como a análise e interpretação do receituário, na qual se fará com base nos aspectos legais e terapêuticos (BRASIL, 2014). Entretanto, visto a carência de Especialistas em Saúde, o fornecimento desses medicamentos, por inúmeras vezes, é feito por técnicos administrativos sob supervisão do profissional farmacêutico.

A região administrativa de Santa Maria apresenta alta vulnerabilidade biopsicossocial e possui apenas duas UBS que realizam o fornecimento de medicamentos psicotrópicos (CODEPLAN, 2018). Desta maneira, há a geração de uma sobrecarga para ambos estabelecimentos de saúde, visto que a dificuldade imposta pela legislação específica é criteriosa. Dentre os empecilhos para um atendimento mais qualificado, cita-se a falta de recursos humanos, carga horária exaustiva, falta de profissional farmacêutico, entre outros, contribuindo para a incidência de erros de fornecimento.

Tais erros podem vir a configurar imperícia, imprudência ou negligência do profissional, principalmente no que tange aos medicamentos psicotrópicos, que agem no Sistema Nervoso Central e tendem a ter estreito índice terapêutico, podendo colocar em risco a saúde física e/ou psicológica do paciente e gerar sérios danos (GOMES, 2018).

Neste sentido, este trabalho tem como objetivo relatar a experiência da elaboração e implementação de estratégias que visam amenizar os erros de fornecimento de medicamentos sujeito a controle especial, utilizando a metodologia da problematização do Arco de Maguerez. 


\section{METODOLOGIA}

Trata-se de um estudo descritivo do tipo relato de experiência realizado na farmácia Unidade Básica de Saúde or de Santa Maria, desenvolvido no período de março a agosto de 202I. Para desenvolvimento da proposta de intervenção, foi utilizada a metodologia de problematização do Arco de Maguerez, que conta com cinco etapas: observação da realidade, os pontos-chave, teorização, hipóteses de solução e aplicação à realidade (BERBEL, 2012). Teve-se como objetivo a diminuição dos erros de fornecimento de psicotrópicos, promovendo assim redução de danos associados ao uso de medicamentos.

Figura I. Trajetória pedagógica para implementação de uma prática educativa problematizadora.

\section{Arco da Problematização de Maguerez}

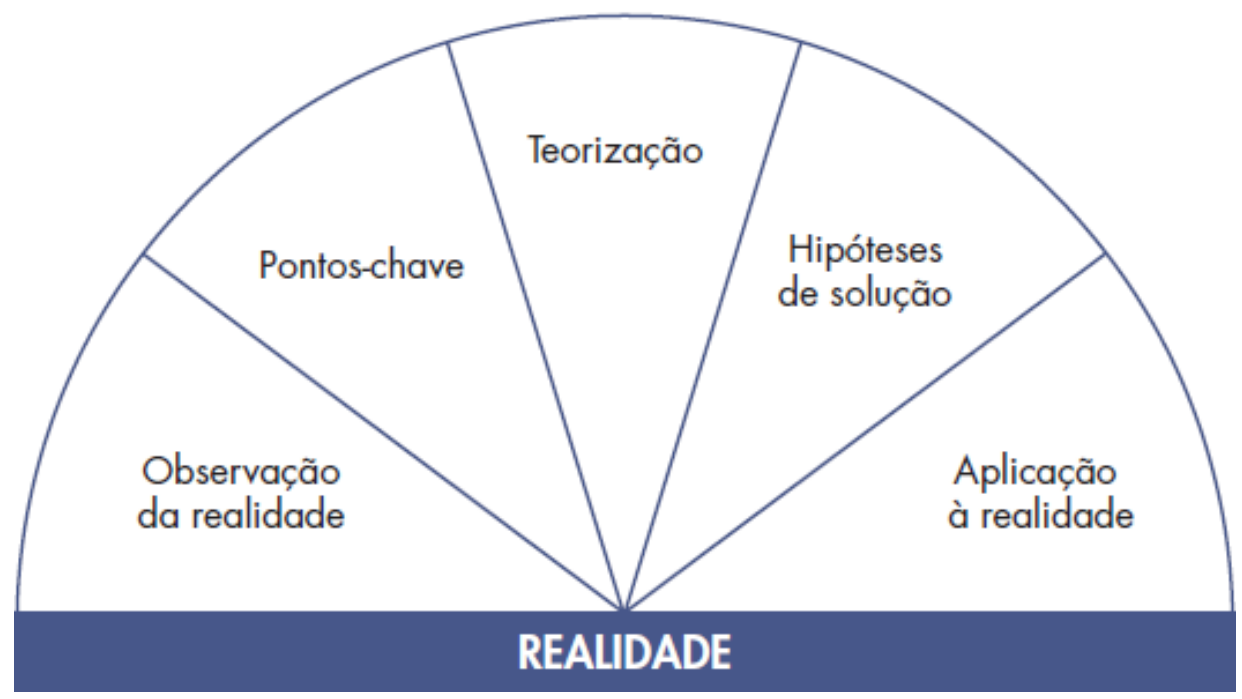

Fonte: Universidade Federal de Santa Catarina, s/d.

\section{Resultados e discussão}

\section{Observação da Realidade}

A partir da observação da rotina e das vivências diárias do local de estudo, notouse que após contagem, havia com certa frequência uma divergência entre o estoque físico e o estoque informatizado de medicamentos psicotrópicos. Prontamente o contato com os pacientes era feito, e após a deteç̧ão de qualquer erro de fornecimento, o mesmo era corrigido. 


\section{Pontos-chave}

Após a observação da realidade e problematização, foi necessário detectar a origem dos erros e o porquê do acontecimento dos mesmos. Os principais pontos observados foram: alta demanda da unidade, levando a exaustão dos técnicos, já que há poucos recursos humanos, aumentando assim a possibilidade da incidência de erros; legislação específica e criteriosa para o fornecimento desses medicamentos, causando confusão e insegurança aos técnicos na hora do fornecimento; e falta de profissional especializado (Especialista em Saúde - Farmacêutico), capaz de supervisionar durante todo o período de atendimento, evitando essas falhas.

\section{Teorização}

Em 2017, reconhecendo o alto risco de danos associados ao uso de medicamentos, a Organização Mundial de Saúde (OMS) lançou o terceiro Desafio Global de Segurança do Paciente com o tema "Medicação sem Danos". A meta desse desafio foi reduzir em 50\% os danos graves e evitáveis relacionados a medicamentos, ao longo de cinco anos, a partir do desenvolvimento de sistemas de saúde mais seguros e eficientes em cada etapa do processo de medicação: prescrição, distribuição, administração, monitoramento e utilização. Para que isso seja possível, foram estabelecidos cinco objetivos específicos focados em ações para reduzir as deficiências nos sistemas de cuidados em saúde, evitando práticas inseguras no uso de medicamentos (ISMP, 2018).

Quadro I. Objetivos do Desafio Global Sobre Segurança de Medicamentos

\begin{tabular}{|c|c|}
\hline AVALIAR & $\begin{array}{c}\text { A natureza e o escopo dos danos evitáveis e fortalecer os sistemas } \\
\text { de monitoramento para detectar e rastrear esses danos. }\end{array}$ \\
\hline CRIAR & $\begin{array}{c}\text { Um plano de ação com foco nos pacientes, profissionais de saúde } \\
\text { e países membros para facilitar a implantação de melhorias na } \\
\text { prescrição, preparo, dispensação, administração e monitoramento } \\
\text { de medicamentos }\end{array}$ \\
\hline DESENVOLVER & $\begin{array}{l}\text { Guias, documentos, tecnologias e ferramentas para dar suporte à } \\
\text { criação de sistemas de utilização de medicamentos mais seguros, } \\
\text { que resultem na diminuição da ocorrência de erros de medicação. }\end{array}$ \\
\hline
\end{tabular}




\begin{tabular}{|c|c|}
\hline ENGAJAR & $\begin{array}{c}\text { Os principais envolvidos, parceiros e indústria para sensibilizá-los } \\
\text { quanto aos problemas de segurança no uso de medicamentos, } \\
\text { levando-os a atuar ativamente em busca de formas de reduzir os } \\
\text { problemas relacionados a medicamentos. }\end{array}$ \\
\hline EMPODERAR & $\begin{array}{c}\text { Pacientes, familiares e cuidadores para que participem ativamente } \\
\text { e de forma engajada nas decisões relacionadas aos seus cuidados } \\
\text { em saúde, fazendo perguntas, identificando erros e gerenciando } \\
\text { ativamente seus medicamentos. }\end{array}$ \\
\hline
\end{tabular}

Fonte: Adaptado de WHO, 2017.

\section{Hipóteses de solução}

Após avaliação da problemática e rastreamento dos pontos-chave foi possível desenvolver estratégias e ferramentas para diminuição da ocorrência dos erros, baseandose nos objetivos estabelecidos pela OMS para a diminuição dos erros de medicação, como: implementação de dupla checagem, criação de ferramentas que aumentam a rastreabilidade de erros e capacitação dos recursos humanos já existentes.

\section{Aplicação à realidade}

- Dupla checagem durante o fornecimento

Com a presença de 3 farmacêuticos na Unidade (Servidor e 2 Profissionais de Saúde Residentes), foi possível criar uma estratégia para que sempre pelo menos i deles estivesse presente durante o fornecimento de medicamentos de controle especial, realizando a dupla checagem dos aspectos técnicos da prescrição e o fornecimento do medicamento. Assim, foi possibilitado o aumento da confiabilidade desse serviço.

- Elaboração do Carimbo Lote/Validade

Foi criado um carimbo, contendo as informações de lote e validade, para ser utilizado em todos os atendimentos das prescrições de controle especial. $O$ carimbo não é de uso obrigatório de acordo com as portarias vigentes, entretanto mostra ser uma excelente ferramenta, aumentando a rastreabilidade do medicamento, caso haja problemas com o lote (DA COSTA MEIRELLES et al., 2015).

Foi possível ainda uma maneira de realizar mais uma checagem, verificando se houve ou não fornecimento do medicamento correto, visto que o lote é anotado durante o 
momento da entrega do medicamento ao paciente. Ao final do dia todas as receitas de controle especial são reanalisadas pelo profissional farmacêutico, quanto a quantidade fornecida, aspectos técnicos da prescrição, e análise do lote anotado, sendo realizada então uma tripla checagem.

- Contagem diária do estoque físico e estoque informatizado

A partir da contagem do estoque físico e conferência com o estoque informatizado, é possível rastrear se houve ou não erros de fornecimento. A contagem física é feita todos os dias na abertura e fechamento do armário que armazena os medicamentos.

- Capacitação dos técnicos administrativos

Não havendo a possibilidade de aumento de recursos humanos, é necessário então a capacitação dos servidores da farmácia. A ação foi realizada mediante dois encontros presenciais com os técnicos administrativos que trabalham na farmácia e são responsáveis pelo fornecimento de medicamentos. As oficinas foram ministradas pelos profissionais farmacêuticos da unidade e trataram sobre assuntos técnicos-gerenciais e clínicoassistenciais diversos.

É importante ressaltar que essa capacitação é um processo de educação continuada, devendo ser constante, visando a adequada atualização desses profissionais. Além disso, destaca-se ainda que o ato se caracteriza como uma forma de apoio matricial, processo de construção de cuidado, estimulado pela Política Nacional de Atenção Básica (BRASIL, 2017).

\section{CONCLUSÃO}

A implementação dessas medidas é fundamental para diminuição dos erros de fornecimento, evitando a ocorrência de possíveis danos aos pacientes ou prejuízos acerca do seu tratamento medicamentoso. Logo no primeiro mês após a execução das intervenções, notou-se bruscamente a redução desses erros. Entretanto destaca-se que é necessária a continuidade do estudo, visando avaliar continuamente os resultados obtidos após a aplicação das propostas de intervenção. 


\section{REFERÊNCIAS}

BERBEL, N. A. N. A metodologia da problematização com o arco de Maguerez: Uma reflexão teórico-epistemológica. Londrina: Eduel, 2012.

BRASIL. Lei no 13.021, de 8 de agosto de 2014. Dispõe sobre o exercício e a fiscalização das atividades farmacêuticas. Diário Oficial da União, Brasília (DF), ago. 20ı4; Seção I.

BRASIL. Ministério da Saúde. Secretaria de Políticas de Saúde. Política nacional de medicamentos. Ministério da Saúde, Secretaria de Políticas de Saúde, Departamento de Atenção Básica. Brasília: Ministério da Saúde, 200I. 40 p.

BRASIL. Portaria $\mathbf{n}^{\circ} 344$, de 12 de maio de 1998. Aprova o Regulamento Técnico sobre substâncias e medicamentos sujeitos a controle especial. Diário Oficial da União, Brasília (DF), dez. 1998.

BRASIL. Portaria no 2.436, de 2I de setembro de 2017. Aprova a Política Nacional de Atenção Básica, estabelecendo a revisão de diretrizes para a organização da Atenção Básica, no âmbito do Sistema Único de Saúde (SUS). Diário Oficial da União, Brasília (DF), set. 2017; Seção I.

BRASÍlLIA. Secretaria de Estado de Saúde. Relação de medicamentos padronizados na Secretaria de Saúde do Distrito Federal: REME-DF. Brasília: Secretaria de Estado de Saúde, 2021.

CODEPLAN. Pesquisa Distrital por Amostra de Domicílios - PDAD 2018. Brasília, 2019. Disponível em: <http://www.codeplan.df.gov.br/wp content/uploads/2020/o6/SantaMaria.pdf $>$. Acesso em: io jun. 202I.

DA COSTA MEIRElleS, C.; OKUMURA, M. T.; SEMMLER, T. C. et al. Gestão da Assistência Farmacêutica: implantação de controle, rastreabilidade e farmacoeconomia. 
Boletim do Instituto de Saúde, v. I6, n. supl., p. 78-82, I nov. 2015.

GOMEZ, S. G. Uso de psicofármacos e as ações da Atenção Básica : uma proposta de intervenção no município de Astorga, Paraná. Tese (pós-graduação) - Curso de Especialização Multiprofissional na Atenção Básica. Universidade Federal de Santa Catarina, Santa $\quad$ Catarina, Disponível em: 〈https://ares.unasus.gov.br/acervo/html/ARES/1327I/I/Sandra_Gonzalez_Gomez.pdf〉. Acesso em: 12 jun. 2021.

ISMP. Instituto Para Práticas Seguras No Uso De Medicamentos. Desafio Global De Segurança Do Paciente Medicação Sem Danos. Belo Horizonte, ISMP Brasil, 2018. Disponível em: <https://www.ismp-brasil.org/site/wpcontent/uploads/2018/o2/ISMP_Brasil_Desafio_Global.pdf>. Acesso em: I5 jun. 2021.

UNA-SUS. Universidade Federal de Santa Catarina. Curso de especialização em Linhas de Cuidado em Enfermagem. Santa Catarina, 2021. Disponível em: 〈https://unasus2.moodle.ufsc.br/course/index.php?categoryid=9〉. Acesso em: I6 jun. 2021.

World Health Organization. Patient Safety. Geneva: World Health Organization, 2017. 